\title{
A Corticostriatal Neural System Enhances Auditory Perception through Temporal Context Processing
}

\author{
Eveline Geiser, Michael Notter, and John D. E. Gabrieli \\ Department of Brain and Cognitive Sciences and McGovern Institute for Brain Research, Massachusetts Institute of Technology, Cambridge, Massachusetts \\ 02139
}

The temporal context of an acoustic signal can greatly influence its perception. The present study investigated the neural correlates underlying perceptual facilitation by regular temporal contexts in humans. Participants listened to temporally regular (periodic) or temporally irregular (nonperiodic) sequences of tones while performing an intensity discrimination task. Participants performed significantly better on intensity discrimination during periodic than nonperiodic tone sequences. There was greater activation in the putamen for periodic than nonperiodic sequences. Conversely, there was greater activation in bilateral primary and secondary auditory cortices (planum polare and planum temporale) for nonperiodic than periodic sequences. Across individuals, greater putamen activation correlated with lesser auditory cortical activation in both right and left hemispheres. These findings suggest that temporal regularity is detected in the putamen, and that such detection facilitates temporal-lobe cortical processing associated with superior auditory perception. Thus, this study reveals a corticostriatal system associated with contextual facilitation for auditory perception through temporal regularity processing.

\section{Introduction}

The temporal structure of acoustic signals is fundamental to their perception. For example, temporal structure influences language parsing (Cutler and Otake, 2002; Frazier et al., 2006) and categorization of acoustic sequences (Hannon and Johnson, 2005). The temporal context of a sequence also crucially affects the perception of elements within that sequence, such as pitch and time intervals (Povel and Okkerman, 1981; Thorpe and Trehub, 1989; Jazayeri and Shadlen, 2010; Borchert et al., 2011; see Sanabria et al., 2011 for a review). If a sound sequence contains temporal regularity, in music also referred to as beat or meter (Lerdahl and Jackendoff, 1983), the sensory processing and the speed of perception of tones concurrent with the regular temporal grid is improved (Barnes and Jones, 2000; Jones et al., 2002, their Experiment 2; Lange, 2009; Ellis and Jones, 2010; Sanabria et al., 2011). Sensory facilitation through temporal regularity is commonly explained by temporal expectation that is derived from the structure of a sound sequence (Barnes and Jones, 2000; London, 2004). Indeed, the anticipation of an event cued by a single static event enhances behavioral performance (Correa et al., 2005;

Received Oct. 12, 2011; revised March 9, 2012; accepted March 14, 2012.

Author contributions: E.G. and J.D.E.G. designed research; E.G. and M.N. performed research; E.G. and M.N. analyzed data; E.G. and J.D.E.G. wrote the paper.

This research was supported by a grant from the Ellison Medical Foundation. E.G. was supported by the Swiss National Science Foundation (PBZHP1-123304). M.N. is currently a student at the University of Zurich. We thank the A. A. Martinos Imaging (enter at the McGovern Institute for Brain Research (Massachusetts Institute of Technology) for assisting in data acquisition, Lyudmila Kushnir for assisting in stimulus construction, and Satra Gosh and Susan Whitfield-Gabrieli for assisting in data analysis.

The authors declare no competing financial interests.

Correspondence should be addressed to Eveline Geiser, Massachusetts Institute of Technology, 46 Vassar Street, Cambridge, MA 02139. E-mail: egeiser@mit.edu.

DOI:10.1523/JNEUROSCI.5153-11.2012

Copyright $\odot 2012$ the authors $\quad 0270-6474 / 12 / 326177-06 \$ 15.00 / 0$
Nobre et al., 2007). Similarly, researchers assume that listeners anticipate the time when future events will occur on the basis of perceived regularity, which in turn facilitates perception (Large and Jones, 1999; Jones et al., 2002). Our study aimed to investigate the neural systems underlying perceptual facilitation in a regular temporal context in human auditory processing.

The perception of temporal regularity has been associated with a thalamic-corticostriatal network that is generally important for timing perception (Mauk and Buonomano, 2004; Buhusi and Meck, 2005; Meck et al., 2008). Specifically, the basal ganglia are associated with temporal regularity perception due to their presumed function in coincidence detection (Matell and Meck, 2004; Buhusi and Meck, 2005; Meck, 2006; Grahn and Brett, 2007; Teki et al., 2011). Cortical areas associated with related timing tasks include supplementary motor cortex, anterior cingulate, and premotor cortex (Chen et al., 2008b; see Coull and Nobre, 2008 for a review; Grahn and McAuley, 2009; Grahn and Rowe, 2009). These studies have shown the importance of the thalamo-corticostriatal network for beat perception on explicit timing tasks (i.e., tasks in which people judge timing per se). However, the neural mechanism by which temporal regularity facilitates auditory perception on implicit timing tasks, where timing is not judged directly but timing influences perceptual performance, is unknown. The implicit influence of timing on perception is likely widespread in audition.

We investigated what neural systems are associated with the facilitation of auditory perception through temporal regularity. Participants made intensity judgments on sound sequences that were temporally regular (periodic) or irregular (nonperiodic). We asked whether temporal regularity would facilitate the accuracy of intensity judgments, and what neural systems were associated with such facilitation. These neural systems may support 


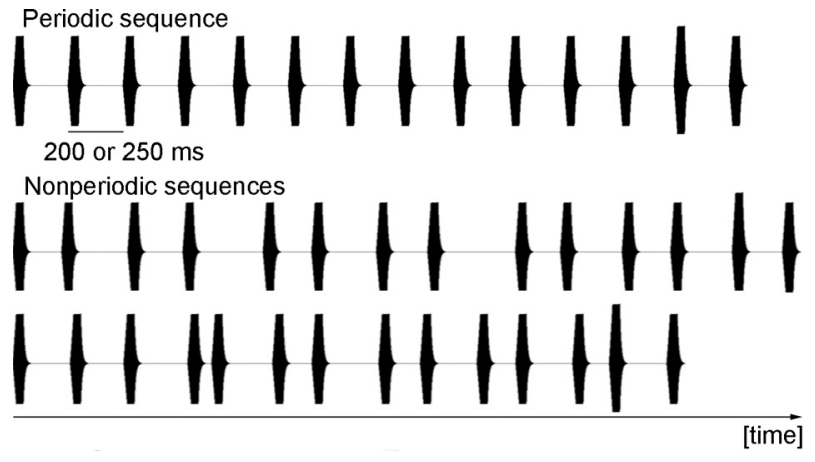

Figure 1. Oscillogram of a periodic (top) and two nonperiodic (bottom) stimulus sequences containing an intensity difference on the 13th and 11th tones, respectively.

the use of temporal regularity to enhance auditory perception in a broad range of auditory process in which temporal regularity facilitates perception.

\section{Materials and Methods}

Participants. Seventeen participants ( 9 men, age $25.1 \pm 4.4$ years) were tested. All participants were right-handed, according to the AnnettHandedness-Questionnaire (Annett, 1992), and had no history of neurological, major medical, psychiatric, or hearing disorders. Participants gave written informed consent for the experiment and were paid for their participation. All procedures were approved by the local ethics committee and were performed in accordance with the revised version of the Declaration of Helsinki.

Stimuli and experimental task. Each stimulus sequence comprised 14 consecutive tones that consisted of a double base frequency of $440 \mathrm{~Hz}$ and three harmonics with half the amplitude of the base frequency. Each tone had a duration of $80 \mathrm{~ms}$ and rise and fall times of 16 and $32 \mathrm{~ms}$, respectively. Fifty percent of the stimulus sequences contained an intensity manipulation in one of tones 11-13. The differences in intensity varied between 115 and $155 \%$ of the base amplitude, as described in the next section. Three intensity differences created three levels of perceptual difficulty, ranging from an easy judgment (high-intensity difference) to a difficult judgment (low-intensity difference). Thus, the experiment adopted a full-factorial model, including experimental conditions of "regularity" (periodic $\times$ nonperiodic) and "intensity" (low-, middle-, high-intensity difference).

The periodic stimulus sequences had intertone-onset intervals of either $200 \mathrm{~ms}$ (50\%) or $250 \mathrm{~ms}(50 \%)$. To construct the nonperiodic stimulus sequences, the intertone-onset intervals were shortened (50\%) and lengthened $(50 \%)$ by values randomly chosen from a uniform distribution and normalized so that the distribution displayed a standard deviation (SD) of $50 \mathrm{~ms}$. The shortened and lengthened intervals were distributed across the sequence in an alternating pattern. A visual example of periodic and nonperiodic stimulus sequences is shown in Figure 1. The stimulus sequences had an average duration of $2.65 \pm 0.12 \mathrm{~s}(50 \%)$ and $3.3 \pm 0.12 \mathrm{~s}(50 \%)$ respectively, which was balanced over the experimental conditions. A total of 288 experimental stimulus sequences and 72 empty trials were presented during 6 experimental sessions of $\sim 8 \mathrm{~min}$ each. Each experimental session was composed of one of three possible task difficulties. The serial order of task difficulties was randomized over the participants, but it always began and ended with the easiest condition to investigate a potential training effect. For each 14-tone sequence, participants had to judge, in a 2-alternative forced choice task, whether any tone differed in intensity from the other tones in the sequence.

Experimental procedure. After obtaining informed consent, participants were instructed and performed 6 practice trials outside of the scanner. While in the scanner, participants performed a behavioral experiment with three different levels of intensity randomly presented $(110,125$, and $150 \%$ of base amplitude). No imaging data were acquired during this behavioral experiment. Based on these behavioral data, we approximately equated participants' sensitivity to intensity differences to choose three levels of intensity (easy: 135-155\%; middle: 125-135\%; difficult: $105-115 \%$ ) for each participant to be presented in the fMRIexperiments. The levels were chosen so that the middle level would approximate a d-prime of 1.5 for each participant.

fMRI acquisition. Structural and functional data were collected on a 3 tesla Siemens scanner equipped with a 32-channel head coil at the Athinoula A. Martinos Imaging Center at McGovern Institute for Brain Research, Massachusetts Institute of Technology. A 3-dimensional highresolution isotropic T1-weighted sequence (TR/TE/T1/flip angle $=2.53$ $\mathrm{s} / 3.48 \mathrm{~ms} / 1.1 \mathrm{~s} / 700)$ provided 176 contiguous slices with $256 \times 256 \mathrm{~mm}$ in plane resolution and a slice thickness of $1 \mathrm{~mm}$ (voxel size $=1 \times 1 \times 1$ $\mathrm{mm}$ ). Functional MRI images were acquired using a standard gradient echo sequence (TR/TE/flip angle $=2 \mathrm{~s} / 30 \mathrm{~ms} / 900)$ that acquired 28 axial functional images with $240 \times 240 \mathrm{~mm}$ plane resolution, $4 \mathrm{~mm}$ slice thickness and a $0.8 \mathrm{~mm}$ interslice gap (voxel size $=2.5 \times 2.5 \times 4.0 \mathrm{~mm}$ ) in ascending order, covering the entire brain. Auditory stimulus sequences were presented through fMRI-compatible insert earphones by Sensimetrics (http://www.sens.com/). Participants' ears were covered with foam ear-defenders for comfort. Stimulus sequences were presented using Presentation software (Neurobehavioral Systems, http://www. neurobs.com/). We implemented a sparse temporal acquisition paradigm collecting one volume every $6 \mathrm{~s}$. Extensive piloting was done to make sure the peak of the BOLD response was covered. The acquisition took place between 4 and $6 \mathrm{~s}$ after the onset of the stimulus sequence.

Behavioral data analysis. For the behavioral data acquired during the fMRI-experiment, the percentage of correct answers was calculated for stimulus sequences either with or without an intensity difference in every experimental condition. The d-prime was calculated for discrimination pairs from each experimental condition. Hit rates of 100 and $0 \%$, respectively, were approximated to two decimal places, resulting in a highest possible d-prime value of 4.6. D-prime values were averaged over all conditions and participants, and they were subjected to a $2 \times 3$ repeatedmeasures ANOVA and post hoc paired $t$ tests.

$f M R I$ analysis. The functional imaging data processing was performed by Nipype (Gorgolewski et al., 2011), using the software package SPM8 (Welcome Department of Cognitive Neurology, London, United Kingdom, http://www.fil.ion.ucl.ac.uk/spm/), Freesurfer (http://surfer.nmr. mgh.harvard.edu/), and FSL (http://www.fmrib.ox.ac.uk/fsl/index. html), unless indicated otherwise. Anatomical data were processed using Freesurfer. The resulting scalp-striped T1-image was later dilated and used for spatial normalization. Functional data were realigned to the first functional volume and were coregistered to individual structural volumes. For spatial smoothing, we applied an isotropic Gaussian kernel of $4 \mathrm{~mm}$ full-width-at-half-maximum. Motion-related artifact detection (threshold 1 SD from the participant mean) and intensity-related artifacts (threshold $=3$ SDs from the participant mean) were identified per participant and excluded from analysis. The statistical evaluation was based on a least-squares estimation using the general linear model for serially autocorrelated observations and was performed separately on each voxel (Friston et al., 1995) in the individual participant's space. Six covariates of interest were included for the conditions of interest. Covariates were convolved with a FIR (Finite Impulse Response) function containing one single basis function and a window length of $2 \mathrm{~s}$, an interscan interval of $8 \mathrm{~s}$ and a microtime resolution of 16 . The serial autocorrelation of the BOLD time series was modeled using a first-order autoregressive mode. The six experimental runs were treated as separate sessions. No global intensity normalization was applied. Low-frequency drifts were removed by including linear and quadratic regressors. No high-pass filter was used. Contrasts of interest on the individual level were calculated and normalized to an averaged anatomical template using ANTs (ANTs Software; Avants et al., 2008; Klein et al., 2009; see Fig. $3)$. Coordinates in Table 1 refer to the approximate region of peak activations on the MNI-T1-template. Random-effects analysis was performed using one-sampled $t$ tests on the second level. Unless otherwise indicated, whole-brain analyses were thresholded at $p<0.001$ and topologically false discovery rate (FDR)-corrected for multiple comparisons on the cluster level at $p<0.05$. Functional ROIs were calculated to characterize effects previously reported on the whole-brain level. ROIs were constructed from the significant activations of the two group con- 


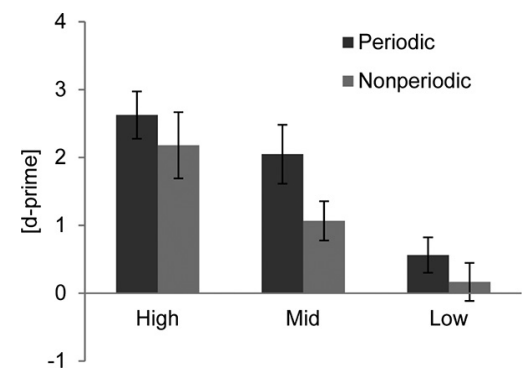

Figure 2. Average d-prime values for high-, middle-, and low-intensity differences plotted separately for periodic (dark) and nonperiodic (light) stimulus sequences. Error bars indicate SE. Discrimination rates significantly differ among the three levels of difficulty $(p<0.001)$ and between periodic and nonperiodic sequences $(p<0.001)$.

Table 1. Stereotactic MNI coordinates and significance levels for periodic > nonperiodic and nonperiodic $>$ periodic contrasts

\begin{tabular}{|c|c|c|c|c|c|c|}
\hline Brain area & Hemisphere & $x$ & $y$ & $z$ & $\begin{array}{l}t \text { value of } \\
\text { cluster peak }\end{array}$ & Cluster size $^{*}$ \\
\hline \multicolumn{7}{|l|}{ Periodic $>$ nonperiodic } \\
\hline Putamen & $\mathrm{R}$ & 22 & 8 & 1 & 4.7 & 250 \\
\hline Putamen** & $\mathrm{L}$ & -21 & 5 & 10 & 4.06 & 192 \\
\hline \multicolumn{7}{|l|}{ Nonperiodic $>$ periodic } \\
\hline HG extending to PT and STG & $\mathrm{R}$ & 67 & -32 & 15 & 7.93 & 4363 \\
\hline HG extending to PT & $\mathrm{L}$ & -42 & -33 & 12 & 6.42 & 4923 \\
\hline
\end{tabular}

trasts (periodic $>$ nonperiodic, nonperiodic $>$ periodic) in the average participants' space. The left homolog of the putamen activation was constructed by applying a voxel-threshold of $p=0.00398$ uncorrected which approximated the size of the right putamen activation in the number of voxels. The four resulting ROIs of the auditory cortices and the putamen were projected back into each individual participant's space. Mean percentage signal changes for the ROIs in the two individual first-level contrasts were extracted for each participant and subjected to Pearson product-moment correlation on the group level. Furthermore, the mean percentage signal changes in the left and right putamen for the periodic $>$ nonperiodic contrast were subjected to a paired-sample $t$ test.

\section{Results}

\section{Behavioral results}

Participants performed the discrimination task significantly better in the periodic $\left(\mathrm{d}^{\prime}=1.5 \pm 0.1\right)$ than the nonperiodic $\left(\mathrm{d}^{\prime}=\right.$ $0.9 \pm 0.2)$ stimulus sequences $\left(F_{(1,16)}=31.8, p<0.001\right.$, Fig. 2$)$. Higher intensity differences were perceived significantly better than lower intensity differences $\left(F_{(2,32)}=46.9, p<0.001\right)$. The discrimination rate was significantly better for the highest intensity $\left(\mathrm{d}^{\prime}=2.1 \pm 0.30\right)$ compared with the middle intensity $\left(\mathrm{d}^{\prime}=\right.$ $\left.1.4 \pm 0.2, F_{(1,16)}=27.2, p<0.001\right)$ and for the middle intensity compared with the low intensity $\left(\mathrm{d}^{\prime}=0.2 \pm 0.2, F_{(1,16)}=38.2\right.$, $p<0.001)$. No significant interaction was observed. There was no significant difference observed between stimulus sequences comprising 200 and $250 \mathrm{~ms}$ intertone-onset interval.

\section{fMRI results}

Activations for periodic and nonperiodic sequences differed reliably in two brain regions, the putamen in the basal ganglia, and auditory regions of the temporal cortex (Table 1, Fig. 3). There was significantly greater activation in the right putamen for periodic compared with nonperiodic sequences. The left homolog of the putamen activation did not surpass the threshold, but did exhibit greater activation at a statistical threshold of $p<0.01$ uncorrected. There was no reliable difference between the right and left putamen ROIs (paired sample $t$ test, $t_{16}=0.83, p=0.42$ ). There was significantly greater activation in bilateral auditory cortices for nonperiodic compared with periodic sequences ( $\mathrm{Ta}-$ ble 1, Fig. 3 middle). This contrast revealed three peaks of activation in the primary auditory cortex (Heschl's gyrus, HG), the lateral temporal gyrus, and the planum temporale (PT). Across individuals, the magnitudes of activation were negatively correlated between the right putamen ROI and right auditory cortex ROI $(r=-0.54, p<0.05)$, and also between the left putamen ROI and left auditory cortex $(r=-0.75, p<0.001)$. Both correlations remained significant after removing from analysis one participant who showed particularly low auditory cortex activation in the context of high putamen activity (Fig. 3). No other significant effects were observed.

\section{Discussion}

Temporal regularity enhanced auditory perception for tone intensity, and there were two associated patterns of brain activation: (1) increased activation for temporally regular (periodic) relative to irregular (nonperiodic) sequences in the putamen, and (2) decreased activation for temporally regular relative to irregular sequences in bilateral auditory regions of the temporal lobes. These two brain regions appeared to be functionally coupled, because across individuals greater activation in left and right putamens was associated with lesser activation in left and right auditory cortical regions, respectively. Thus, the present finding suggests that temporal regularity facilitates perception by an interaction between a striatal mechanism encoding temporal regularity, and a cortical mechanism that performs the perceptual analysis, such as intensity detection, that is supported by the regularity.

\section{Behavioral results}

The novel finding that temporal regularity facilitates auditory intensity discrimination is consistent with reports that temporal regularity facilitates other dimensions of auditory perception, including temporal cue perception (Large and Jones, 1999; Barnes and Jones, 2000; McAuley and Miller, 2007; Correa et al., 2009; Ellis and Jones, 2010; Sanabria et al., 2011) and pitch perception (Jones et al., 2002). This suggests that the temporal-regularity facilitation of auditory perception may generalize broadly to other auditory dimensions. The facilitation of perception by temporal regularity was robust, with 14 of 17 participants showing behavioral facilitation at all three levels of task difficulty (the 3 other participants showing facilitation at one level). In general, listeners display a preference for periodic over nonperiodic sequences (Martin, 1972; Handel and Lawson, 1983; Essens and Povel, 1985; Essens, 1986; Collier and Wright, 1995; McDermott and Hauser, 2005; Patel et al., 2005). This preference may be associated with enhanced ease of perceptual analysis.

\section{Functional imaging results}

Enhanced auditory perception for intensity was associated with activations in the basal ganglia (greater for periodic sequences) and auditory regions of the temporal lobes (greater for nonperiodic sequences). The opposite pattern of activation in the two brain regions suggests that the activations were not simply correlates of task difficulty, but rather reflected cooperating and functionally distinct striatal and cortical mechanisms that exploited temporal regularity so as to enhance perceptual accuracy. 
The thalamo-corticostriatal system is known to be involved in timing and temporal regularity perception (Matell and Meck, 2004; Mauk and Buonomano, 2004; Buhusi and Meck, 2005; Meck, 2006; Chen et al., 2008b; Meck et al., 2008). Studies focusing on beat-based, or temporally regular, timing perception have found activation in the striatum for regular temporal processing (Grahn and Brett, 2007). In our study, striatal activation was confined to the putamen, as in some studies (Grahn and Brett, 2007; Grahn and Rowe, 2009), whereas other studies have reported activation in both putamen and caudate (Teki et al., 2011). Prior studies of beat-based timing examined explicit timing tasks involving direct judgments about or attention to timing per se, such as regularity detection (Grahn and Rowe, 2009), duration discrimination (Teki et al., 2011), rhythm reproduction or beat synchronization (Riecker et al., 2003; Chen et al., 2008a), or simply instruction to listen to the beat (Grahn and Brett, 2007). In contrast, the present study involved a judgment about intensity, and temporal regularity was used implicitly to support intensity discrimination. Explicit judgments on temporal regularity are likely rare relative to many situations in which the focus of attention is not the temporal regularity of the auditory input, but rather perception of the content of the input. Thus, the present study indicates that the striatum encodes temporal regularity even when that regularity is not the object of attention. This expands the potential role of the striatum in using temporal regularity to enhance perception.

In the present study, only the right putamen exhibited reliable activation for periodic relative to nonperiodic stimulus sequences, but the same kind of activation was evident at a more liberal threshold in the left putamen, there was no significant difference between left and right putamen activation, and there were comparable negative correlations across participants in left and right hemispheres between putamen and auditory cortical activations. Therefore, it seems likely that the activation was essentially bilateral and not reflecting hemispheric specialization.

Temporal regularity, and its related superior auditory performance, was associated with reduced activations in primary and secondary auditory cortices in both hemispheres, namely, the Heschl's gyrus and the planum temporal. Superior performance in auditory tasks is sometimes associated with increased or expanded fMRI signals, as in studies investigating musical expertise (Ohnishi et al., 2001; Elmer et al., 2011) or auditory training (Callan et al., 2003). However, other studies report the opposite effect: decreased auditory cortex activation related to superior performance after auditory training (Jäncke et al., 2001b; Erickson et al., 2007). Enhanced performance due to repetition also correlates with reduced cortical activation, or repetition suppression, in auditory cortex (Bergerbest et al., 2004; De Lucia et al., 2010; Doehrmann et al., 2010). Thus, our study is aligned with prior reports indicating reduced BOLD signal in sensory cortices can be associated with superior or more efficient perceptual performance.

One interpretation of the findings is that the encoding of temporal regularity in the putamen reduced the demands of auditory analysis in temporal cortex, resulting in both more accurate perception and less activation. The finding that across participants, in both hemispheres, greater putamen activation for periodic sequences correlated with lesser cortical activation for those sequences supports this possibility. Although the experimental design cannot establish a causal relation between the two regions of activation, it appears most likely that reduced activation for periodic sequences in auditory cortex reflects reduced processing demands for the periodic sequences.

Superior performance and decreased auditory cortical activation could reflect an attentional mechanism guided by temporal expectation. The dynamic-attending theory posits that a temporally regular, periodic context facilitates perception through stimulus sequence-induced entrainment of temporal expectation, resulting in periodic increase and decrease of attention (Large and Jones, 1999; Barnes and Jones, 2000). Similarly, temporal expectation induced by priming or stimulus-driven predictability results in perceptual facilitation reflected in decreased reaction times (Nobre et al., 2007). Our participants were required to listen carefully to each tone of a sequence to perform the task. Consequently, anticipating the time point when a tone would appear could facilitate this process, and entrainment would reduce demands on attention when processing periodic acoustic sequences. 
Prior imaging studies examining regular versus irregular auditory stimuli did not find greater activation in auditory cortex for irregular stimuli (Geiser et al., 2008; Grahn and McAuley, 2009). Those studies, however, used tasks that required detection of whether a sequence was regular; nonregular sequences could be identified at the earliest moment temporal irregularity was detected, whereas regular sequences required attention to the entire sequence to make certain that no irregularity was included. Lengthier attention to regular sequences could have obscured any reduction in activation associated with regularity. Thus, one interpretation of our results is that reduced cortical activation reflects reduced attention in making a perceptual decision. Indeed, manipulation of attention has been shown to influence auditory cortex activation. Increased attention resulted in increased cortical activation (Jäncke et al., 2001a; Rinne et al., 2005), specifically in the secondary auditory cortex (Griffiths and Warren, 2002; Jäncke et al., 2002; Ahveninen et al., 2006, 2011; Brechmann et al., 2007). Thus, reduced activation in the auditory cortex in the present study could reflect reduced attentional demands during periodic sequence processing.

Enhanced intensity detection for periodic sequences could result from the coincidence of temporal processing and auditory perception. Electrophysiological findings suggest a neural mechanism by which implicit timing perception induces perceptual facilitation. Spontaneous rhythmic activity at $1-4 \mathrm{~Hz}$ (delta activity), measured through intercranial microelectrodes in sensory cortices of nonhuman primates or in the human electroencephalogram, modulates excitability of cortical neurons and, consequently, sensitivity to external stimuli (Bishop, 1932; Buzsáki and Chrobak, 1995; Lakatos et al., 2005; Stefanics et al., 2010). Influenced by attention and task relevant stimulus properties including temporal predictability, this spontaneous activity can be synchronized to an external stimulus, thus influencing neuronal excitability (Lakatos et al., 2005, 2009; Besle et al., 2011). These electrophysiological studies measured neural activity in auditory cortex, and the present study, which found activation for temporal regularity in the putamen, suggests that a source of influence on auditory cortex could be a synchronization process within thalamo-corticostriatal loops.

The present findings suggest that there is a cortical-striatal system that enhances auditory perception through temporal context processing. The bilateral and negative correlation between putamen and auditory-cortical activations suggests that stronger representation of temporal regularity in the striatum reduces the processing burden of auditory cortex. While we cannot determine a causal direction between activations in the putamen and in the auditory cortex, this finding indicates a functional relation between striatal and auditory cortex activations. Anatomical connections between the striatum and the superior temporal region found in nonhuman primates (Yeterian and Pandya, 1998; Borgmann and Jürgens, 1999) could mediate this functional relation between striatum and cortex that enhances auditory perception on the basis of temporal regularity.

\section{References}

Ahveninen J, Jääskeläinen IP, Raij T, Bonmassar G, Devore S, Hämäläinen M, Levänen S, Lin FH, Sams M, Shinn-Cunningham BG, Witzel T, Belliveau JW (2006) Task-modulated "what" and "where" pathways in human auditory cortex. Proc Natl Acad Sci U S A 103:14608-14613.

Ahveninen J, Hämäläinen M, Jääskeläinen IP, Ahlfors SP, Huang S, Lin FH, Raij T, Sams M, Vasios CE, Belliveau JW (2011) Attention-driven auditory cortex short-term plasticity helps segregate relevant sounds from noise. Proc Natl Acad Sci U S A 108:4182-4187.

Annett M (1992) Five tests of hand skill. Cortex 28:583-600.
Avants BB, Epstein CL, Grossman M, Gee JC (2008) Symmetric diffeomorphic image registration with cross-correlation: evaluating automated labeling of elderly and neurodegenerative brain. Med Image Anal 12:26-41.

Barnes R, Jones MR (2000) Expectancy, attention, and time. Cognit Psychol 41:254-311.

Bergerbest D, Ghahremani DG, Gabrieli JD (2004) Neural correlates of auditory repetition priming: reduced fMRI activation in the auditory cortex. J Cogn Neurosci 16:966-977.

Besle J, Schevon CA, Mehta AD, Lakatos P, Goodman RR, McKhann GM, Emerson RG, Schroeder CE (2011) Tuning of the human neocortex to the temporal dynamics of attended events. J Neurosci 31:3176-3185.

Bishop G (1932) Cyclic changes in excitability of the optic pathway of the rabbit. Am J Physiol 103:213-224.

Borchert EM, Micheyl C, Oxenham AJ (2011) Perceptual grouping affects pitch judgments across time and frequency. J Exp Psychol Hum Percept Perform 37:257-269.

Borgmann S, Jürgens U (1999) Lack of cortico-striatal projections from the primary auditory cortex in the squirrel monkey. Brain Res 836:225-228.

Brechmann A, Gaschler-Markefski B, Sohr M, Yoneda K, Kaulisch T, Scheich H (2007) Working memory specific activity in auditory cortex: potential correlates of sequential processing and maintenance. Cereb Cortex 17:2544-2552.

Buhusi CV, Meck WH (2005) What makes us tick? Functional and neural mechanisms of interval timing. Nat Rev Neurosci 6:755-765.

Buzsáki G, Chrobak JJ (1995) Temporal structure in spatially organized neuronal ensembles: a role for interneuronal networks. Curr Opin Neurobiol 5:504-510.

Callan DE, Tajima K, Callan AM, Kubo R, Masaki S, Akahane-Yamada R (2003) Learning-induced neural plasticity associated with improved identification performance after training of a difficult second-language phonetic contrast. Neuroimage 19:113-124.

Chapin HL, Zanto T, Jantzen KJ, Kelso SJ, Steinberg F, Large EW (2010) Neural responses to complex auditory rhythms: the role of attending. Front Psychol 1:224.

Chen JL, Penhune VB, Zatorre RJ (2008a) Moving on time: brain network for auditory-motor synchronization is modulated by rhythm complexity and musical training. J Cogn Neurosci 20:226-239.

Chen JL, Penhune VB, Zatorre RJ (2008b) Listening to musical rhythms recruits motor regions of the brain. Cereb Cortex 18:2844-2854.

Collier GL, Wright CE (1995) Temporal rescaling of simple and complex ratios in rhythmic tapping. J Exp Psychol Hum Percept Perform 21:602-627.

Correa A, Lupiáñez J, Tudela P (2005) Attentional preparation based on temporal expectancy modulates processing at the perceptual level. Psychon Bull Rev 12:328-334.

Correa A, Rao A, Nobre AC (2009) Anticipating conflict facilitates controlled stimulus-response selection. J Cogn Neurosci 21:1461-1472.

Coull J, Nobre A (2008) Dissociating explicit timing from temporal expectation with fMRI. Curr Opin Neurobiol 18:137-144.

Cutler A, Otake T (2002) Rhythmic categories in spoken-word recognition. J Mem Lang 46:296-322.

De Lucia M, Cocchi L, Martuzzi R, Meuli RA, Clarke S, Murray MM (2010) Perceptual and semantic contributions to repetition priming of environmental sounds. Cereb Cortex 20:1676-1684.

Doehrmann O, Weigelt S, Altmann CF, Kaiser J, Naumer MJ (2010) Audiovisual functional magnetic resonance imaging adaptation reveals multisensory integration effects in object-related sensory cortices. J Neurosci 30:3370-3379.

Ellis RJ, Jones MR (2010) Rhythmic context modulates foreperiod effects. Atten Percept Psychophys 72:2274-2288.

Elmer S, Meyer M, Marrama L, Jäncke L (2011) Intensive language training and attention modulate the involvement of fronto-parietal regions during a non-verbal auditory discrimination task. Eur J Neurosci 34:165-175.

Erickson KI, Colcombe SJ, Wadhwa R, Bherer L, Peterson MS, Scalf PE, Kim JS, Alvarado M, Kramer AF (2007) Training-induced functional activation changes in dual-task processing: an FMRI study. Cereb Cortex 17:192-204.

Essens PJ (1986) Hierarchical organization of temporal patterns. Percept Psychophys 40:69-73.

Essens PJ, Povel DJ (1985) Metrical and nonmetrical representations of temporal patterns. Percept Psychophys 37:1-7. 
Frazier L, Carlson K, Clifton C Jr (2006) Prosodic phrasing is central to language comprehension. Trends Cogn Sci 10:244-249.

Friston KJ, Holmes AP, Poline JB, Grasby PJ, Williams SC, Frackowiak RS, Turner R (1995) Analysis of fMRI time-series revisited. Neuroimage 2:45-53.

Geiser E, Zaehle T, Jancke L, Meyer M (2008) The neural correlate of speech rhythm as evidenced by metrical speech processing. J Cogn Neurosci 20:541-552.

Gorgolewski K, Burns CD, Madison C, Clark D, Halchenko YO, Waskom ML, Ghosh SS (2011) Nipype: a flexible, lightweight and extensible neuroimaging data processing framework in python. Front Neuroinform 5:13.

Grahn JA, Brett M (2007) Rhythm and beat perception in motor areas of the brain. J Cogn Neurosci 19:893-906.

Grahn JA, McAuley JD (2009) Neural bases of individual differences in beat perception. Neuroimage 47:1894-1903.

Grahn JA, Rowe JB (2009) Feeling the beat: Premotor and striatal interactions in musicians and nonmusicians during beat perception. J Neurosci 29:7540-7548.

Griffiths TD, Warren JD (2002) The planum temporale as a computational hub. Trends Neurosci 25:348-353.

Handel S, Lawson GR (1983) The contextual nature of rhythmic interpretation. Percept Psychophys 34:103-120.

Hannon EE, Johnson SP (2005) Infants use meter to categorize rhythms and melodies: implications for musical structure learning. Cognit Psychol 50:354-377.

Jäncke L, Buchanan TW, Lutz K, Shah NJ (2001a) Focused and nonfocused attention in verbal and emotional dichotic listening: An FMRI study. Brain Lang 78:349-363.

Jäncke L, Gaab N, Wüstenberg T, Scheich H, Heinze HJ (2001b) Short-term functional plasticity in the human auditory cortex: an fMRI study. Brain Res Cogn Brain Res 12:479-485.

Jäncke L, Wüstenberg T, Scheich H, Heinze HJ (2002) Phonetic perception and the temporal cortex. Neuroimage 15:733-746.

Jazayeri M, Shadlen MN (2010) Temporal context calibrates interval timing. Nat Neurosci 13:1020-1026.

Jones MR, Moynihan H, MacKenzie N, Puente J (2002) Temporal aspects of stimulus-driven attending in dynamic arrays. Psychol Sci 13:313-319.

Klein A, Andersson J, Ardekani BA, Ashburner J, Avants B, Chiang MC, Christensen GE, Collins DL, Gee J, Hellier P, Song JH, Jenkinson M, Lepage C, Rueckert D, Thompson P, Vercauteren T, Woods RP, Mann JJ, Parsey RV (2009) Evaluation of 14 nonlinear deformation algorithms applied to human brain MRI registration. Neuroimage 46:786-802.

Lakatos P, Shah AS, Knuth KH, Ulbert I, Karmos G, Schroeder CE (2005) An oscillatory hierarchy controlling neuronal excitability and stimulus processing in the auditory cortex. J Neurophysiol 94:1904-1911.

Lakatos P, O'Connell MN, Barczak A, Mills A, Javitt DC, Schroeder CE (2009) The leading sense: supramodal control of neurophysiological context by attention. Neuron 64:419-430.

Lange K (2009) Brain correlates of early auditory processing are attenuated by expectations for time and pitch. Brain Cogn 69:127-137.
Large EW, Jones MR (1999) The dynamics of attending: How people track time-varying events. Psychol Rev 106:119-159.

Lerdahl F, Jackendoff R (1983) An overview of hierarchical structure in music. Music Percept 1:229-252.

London JM (2004) Hearing in time: psychological aspects of musical meter. New York: Oxford UP.

Martin JG (1972) Rhythmic (hierarchical) versus serial structure in speech and other behavior. Psychol Rev 79:487-509.

Matell MS, Meck WH (2004) Cortico-striatal circuits and interval timing: coincidence detection of oscillatory processes. Brain Res Cogn Brain Res $21: 139-170$.

Mauk MD, Buonomano DV (2004) The neural basis of temporal processing. Annu Rev Neurosci 27:307-340.

McAuley JD, Miller NS (2007) Picking up the pace: effects of global temporal context on sensitivity to the tempo of auditory sequences. Percept Psychophys 69:709-718.

McDermott J, Hauser M (2005) The origins of music: innateness, uniqueness, and evolution. Music Percept 23:29-59.

MeckWH (2006) Neuroanatomical localization of an internal clock: a functional link between mesolimbic, nigrostriatal, and mesocortical dopaminergic systems. Brain Res 1109:93-107.

Meck WH, Penney TB, Pouthas V (2008) Cortico-striatal representation of time in animals and humans. Curr Opin Neurobiol 18:145-152.

Nobre A, Correa A, Coull J (2007) The hazards of time. Curr Opin Neurobiol 17:465-470.

Ohnishi T, Matsuda H, Asada T, Aruga M, Hirakata M, Nishikawa M, Katoh A, Imabayashi E (2001) Functional anatomy of musical perception in musicians. Cereb Cortex 11:754-760.

Patel AD, Iversen JR, Chen Y, Repp BH (2005) The influence of metricality and modality on synchronization with a beat. Exp Brain Res 163:226-238.

Povel DJ, Okkerman H (1981) Accents in equitone sequences. Perception and Psychophysics 30:565-572.

Riecker A, Wildgruber D, Mathiak K, Grodd W, Ackermann H (2003) Parametric analysis of rate-dependent hemodynamic response functions of cortical and subcortical brain structures during auditorily cued finger tapping: a fMRI study. Neuroimage 18:731-739.

Rinne T, Pekkola J, Degerman A, Autti T, Jääskeläinen IP, Sams M, Alho K (2005) Modulation of auditory cortex activation by sound presentation rate and attention. Hum Brain Mapp 26:94-99.

Sanabria D, Capizzi M, Correa A (2011) Rhythms that speed you up. J Exp Psychol Hum Percept Perform 37:236-244.

Stefanics G, Hangya B, Hernádi I, Winkler I, Lakatos P, Ulbert I (2010) Phase entrainment of human delta oscillations can mediate the effects of expectation on reaction speed. J Neurosci 30:13578-13585.

Teki S, Grube M, Kumar S, Griffiths TD (2011) Distinct neural substrates of duration-based and beat-based auditory timing. J Neurosci 31:3805-3812.

Thorpe LA, Trehub SE (1989) Duration illusion and auditory grouping in infancy. Dev Psychol 25:122-127.

Yeterian EH, Pandya DN (1998) Corticostriatal connections of the superior temporal region in rhesus monkeys. J Comp Neurol 399:384-402. 